\title{
THE MRC 1-JY SAMPLE OF RADIO GALAXIES
}

\author{
V. K. KAPAHI ${ }^{1}$, P. J. MCCARTHY ${ }^{2}$, R. M. ATHREYA ${ }^{1}$ \\ ${ }^{1}$ NCRA-TIFR, P.Bag 3, Ganeshkhind, Pune - 411007, India \\ 2 OCIW, 813 Santa Barbara St., Pasadena, Ca 91101, USA
}

\author{
AND \\ W. VAN BREUGEL ${ }^{3}$, C. R. SUBRAHMANYA ${ }^{1}$ \\ ${ }^{3}$ IGPP, Lawrence Livermore Lab., Livermore, Ca 94550, USA
}

Most known galaxies at high redshifts have been found by concentrating on ultra steep spectrum $(\alpha \gtrsim 1)$ sources in low frequency radio surveys (eg.Rottgering et al., 1994; McCarthy et al., 1990). We have been carrying out a systematic study of a large and complete sample from the $408 \mathrm{MHz}$ Molonglo Reference Catalogue which is not biased by any spectral index criterion. Containing 558 sources, defined by $S_{408} \geq 0.95 \mathrm{Jy} ;-30^{\circ}<\delta<-20^{\circ}$ and the RA ranges of $20^{h} 20^{m}$ to $06^{h} 00^{m}$ and $09^{h} 20^{m}$ to $14^{h} 00^{m}$, our sample is about 3 times larger in size and 5 times deeper in flux density than the well studied 3CRR sample.

Observations and Current Status : To obtain complete optical identifications we have mapped all the sources using the VLA at $6 \mathrm{~cm} \lambda$ with a resolution of $\sim 1$ " to 5 " arc and made CCD images in the r-band using the 1-m and 2.5-m telescopes of the Las Campanas Observatory. We have also imaged a majority of the sources in the K-band. While about $95 \%$ of the sources are identified down to $\mathrm{r}=25, \sim 99 \%$ get identified down to $\mathrm{K} \sim 19$. Spectroscopic redshifts for the sample are being measured at Las Campanas and with the 4-m CTIO telescope at Cerro Tololo.

Apart from the 111 objects forming a complete sample of quasars (including $6 \mathrm{BL}$ Lac objects), we have 447 radio galaxies of which $\sim 60 \%$ have spectroscopic redshifts. Approximate redshifts for another $22 \%$ of the sample can be estimated using the $\mathrm{K}-\mathrm{z}$ relation. Thus only $\sim 18 \%$ of the galaxies lack a measured or estimated $\mathrm{z}$ and this is largely due to shortage of telescope time at some right ascensions. The available redshifts are therefore likely to be representative of the whole sample. The median redshift of 


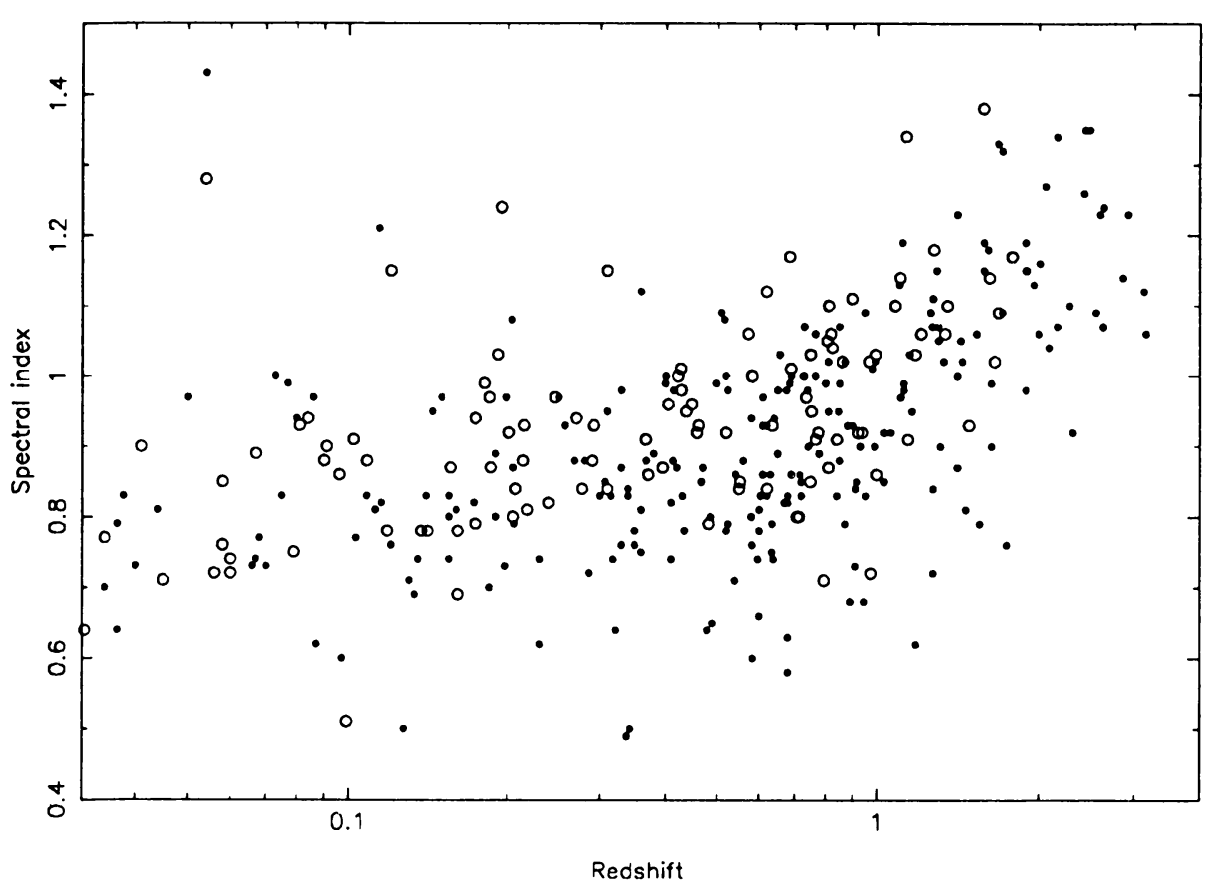

Figure 1. The spectral index - redshift plot for radio galaxies in the MRC (filled circles) and 3CRR (open circles) samples.

the galaxies is $\sim 0.8$, much larger than the median value of $\sim 0.29$ for the 3CRR galaxies.

Spectral Index - Redshift Relation : The value of $\alpha_{0.4}^{4.86}$ (between $408 \mathrm{MHz}$ and $4.86 \mathrm{GHz}$ ) for all galaxies in the Molonglo Sample with a measured or estimated $\mathrm{z}$ is plotted against $\mathrm{z}$ in Figure 1 together with the corresponding data for the $3 \mathrm{CRR}$ galaxies. A preliminary analysis indicates that $\alpha$ is more likely to correlate with $\mathrm{z}$ than with radio luminosity. We also find that most of the $\alpha-z$ correlation could arise from just the cosmological $\mathrm{K}$-correction, as most of the Molonglo galaxies at $\mathrm{z}>2$ have been observed to have spectra that become progressively steeper going from $408 \mathrm{MHz}$ to $\sim 8 \mathrm{GHz}$ (Athreya et al. 1996).

\section{References}

Athreya et al. (1996) Radio Polarization studies of galaxies at $\mathrm{z}>2$ - This volume. McCarthy, P.J. et al. (1990) A.J., Vol. 100, 1014.

Rottgering, A.J.A. et al. (1994) Astron. Astrophys. Suppl. Ser ., Vol.108, 79. 